International Journal of Computer Science \& Information Technology (IJCSIT), Vol 3, No 2, April 2011

\title{
A DESIGN OF A COMPUTER-AIDED DIAGNOSTIC TOOL FOR CHEST X-RAY ANALYSIS
}

\author{
Kim Le \\ Faculty of Information Sciences and Engineering, University of Canberra \\ University Drive, Bruce, ACT-2601, AUSTRALIA \\ kim. ledcanberra.edu. au
}

\begin{abstract}
Diagnosis of some chest diseases like heart failure, lung cancer or lung tuberculosis, etc. is often based on chest X-ray images (CXR). The diseases are curable if they are detected in their early stages. Examining CXR is a time consuming process. In some cases, medical experts had overlooked the diseases in their firstexaminations on $C X R$, and when the images were reexamined, the disease signs could be detected.Furthermore, the number of CXR to examine is numerous and far beyond the capability of available medical staff, especially in developing countries.
\end{abstract}

A computer-aided diagnosis (CAD) system can mark suspected areas on CXR for careful examination by medical doctors, and can give alarm in the cases that need urgent attention.

This paper reports our continuous work on the development of a CAD tool, which is an extended version of a conference paper [1]. In this version, we present some more works on CXR analysis and examination.

\section{KEYWORDS}

Computer aided diagnosis; Automated disease diagnosis; Chest X-ray analysis; Watershed segmentation; Cancer, tuberculosis and heart failure diseases.

\section{INTRODUCTION}

Heart failure is a very serious disease, and early detection of its symptom is of vital importance. A normal heart may become serious sick just several months later. The treatment of lung cancer and tuberculosis (TB) is easier in their early stages but very difficult in the advanced stages of the diseases. The overall 5-year survival rate for lung cancer patients increases from 14 to $49 \%$ if the disease is detected in time [2,3]. Although Computed Tomograph (CT) can be more efficient than X-ray [3], the latter is more generally available. Therefore preliminary diagnosis for TB and lung cancer, currently performed by medical doctors, is mainly based on chest Xray images (CXR). This is a time-costly process, and the quantity of images to be examined is at an unmanageable level, especially in populous countries with scarce medical professionals.

Computerised analysis of CXR images can reveal chest diseases in their early stages. An early symptom of congestive heart failure is the increase of the cardiothoracic ratio when it is approaching a limit. Most cancer and TB cases start with the appearance of small nodules, 
which are hard to detect at first examinations. Our current work aims at the design and implementation of an automated X-ray image analyser to detect early signs of some chest diseases.

When radiologists examine a CXR image, they first need to recognise the two lungs and then find any obvious abnormality. Hence CXR segmentation is an essential process. Image segmentation is often based on the Watershed method [4] or the energy-minimization technique. The Watersnakes method [5], which uses the Watershed as a starting point, tries to make a link between the two segmentation approaches with the introduction of an adjustable energy function to change the smoothness of the boundary of a segmented area. However the Watersnakes method may be not suitable for lung segmentation on CXR.

This paper reports our continuous work on the development of a CAD tool for detection of early symptoms of some chest diseases. The paper is organised as follows. Section 2 presents a brief introduction to CXR analysis in the viewpoints of radiologists. In Section 3, we propose a Watershed-based method for lung segmentation. Once lung objects have been isolated, early symptoms of some chest diseases like heart failure, lung cancer and TB, lung collapse, etc. will be identified. The paper ends with a brief discussion.

\section{CHEST X-RAY ANALYSIS}

CXR analysis is a basic task in medicine but it is a complex task based on careful observation, sound anatomical principles, and knowledge of physiology and pathology [6]. PA (posterioranterior) and lateral chest X-ray images are often read together, and they complement each another. The PA exam is viewed as if the patient is standing in front of the examiner; hence the patient's right lung is on the left of a CXR. In our current work, we focus only on PA.

The basic diagnostic task is to detect abnormality. To isolate lung objects, radiologists need to know both the structures within the mediastinum forming the mediastinal margins and the lobes of the lungs forming the margins of the lungs along the mediastinum and chest wall. Some abnormality may be recognised easily, but some may need careful examination as well as accurate measurement. For example, the detection of an early symptom of congestive heart failure needs the measure of the thoracic diameter. Lung cancer and TB start with small nodules, etc. which are hard to detect. Comparison different CXR taken in some regular examinations can be invaluable, and radiologists often need to observe some CXR at the same time for comparison.

\section{CHEST X-RAY IMAGE PROCESSING}

The first task for CXR analysis is to isolate lungs from the background. Different techniques can be used to find lung boundaries. Once the lung objects have been isolated, the CXR image is analysed to detect abnormalities.

\subsection{Lung Isolation}

On an X-ray image, the gray levels of pixels, ranging from 0 (black) to 255 (white), depend on both the thickness of tissues and their atomic weights, and they are clustered in the middle range of gray levels, and those of air and bone pixels are in the two extremes, black and white, respectively. In the case of a CXR image, the two lungs are darker than the background, and are easy to recognise. However the existence of ribs, shoulders and pulmonary vessels with higher gray levels makes lung boundaries harder to detect accurately (Figure 1a). 
The Watershed segmentation was originally used in topography to partition an area into regions. A Watershed segmentation process starts at some regional minima LI, the lowest points in the area that water can flow into. The area is divided into some regions VI (valleys) that are grown from the corresponding minima LI by adding to VI, iteratively, unlabelled higher points on their boundaries. The addition is repeated until no more point can be assigned to any region.

In the case of a CXR image, gray levels play the role as that of ground levels in topography. The Watershed segmentation can be used to isolate the two lungs and the dark background. However the existence of the bright region between the two lungs and the bright regions of ribs, shoulders, etc. make the original Watershed segmentation unable to be stopped accurately at the lung boundaries. We propose two modifications:

a) In addition to the minima $\mathrm{LI}$, we also find some maxima $\mathrm{HJ}$, the highest points. The regions that originally consist of these maxima are called MJ (mountain). The mountain regions are grown, in concurrence with the growing of the valleys, by adding to MJ unlabelled lower points on their boundaries. The modified Watershed segmentation is carried on with the growing of all valleys VI and mountains MJ. The segmentation is completed when there is no more point can be added to any region.

b) When the modified Watershed segmentation is complete. The boundaries between a lung and the mediastinum may be too far within the mediastinum. A drying process, starting from the maxima, will push the lung boundary back towards the lungs' cores.

The Watershed-based segmentation to find lungs' boundaries of a CXR image is summarised and is illustrated as follows.

\section{Lung Boundary Detection Algorithm:}

a) Find the gray level histogram for the CXR.

b) Based on the histogram, choose six gray levels GL(i), i $=0,1, \ldots, 5$, which are used to sort he pixels of the CXR into five regions Region $(\mathrm{j}), \mathrm{j}=0, \ldots, 4$, each with a specific percentage of pixels $\mathrm{P}(\mathrm{j})$, e.g. $20 \%, 10 \%, 20 \%, 20 \%$ and $30 \%$, with $\mathrm{P}(0)=20 \%$ for Region (0), $\mathrm{P}(1)=10 \%$ for Region (1), etc., so that

$$
\forall \text { pixels } \in \text { Region }(\mathrm{j}), \mathrm{j}=0, . .4 \text {, pixel.grayLevel } \in(G L(j), G L(j+1))
$$

Where GL $(0)=0$ and GL $(5)=255$, the minimal and the maximal gray levels respectively.

The lung cores (valleys) including pixels with gray levels less than GL (1) then should be in Region (0) (Figure 1b). Dark pixels on the narrow strips along the left and the right sides of the CXR image (Figure 1c) also belong to Region (0). Brighter pixels (mountains) in a short vertical strip at the middle of the image belong to Region (3) or Region (4).

To differentiate dark pixels of lung cores from dark pixels of the background, we divide the CXR into $4 \times 4$ rectangles $R(i, j), i, j=0,1,2 \& 3$.. The dark pixels lying in the four centralrectangles, i.e. $\mathrm{R}(\mathrm{m}, \mathrm{n}), \mathrm{m}, \mathrm{n}=1,2$, should belong to lung cores, and are labelled with +1 .The dark pixels on the strips along the left and the right sides of CXR should belong to the background and are labelled with -1 . The bright pixels in the vertical strip mentioned above should belong to the background and are labelled with 2 . 
These dark and brighter pixels of the background (with labels -1 or -2 ), as well as dark pixels of the lung cores (with labelled +1 ) are used as growing seeds in the next step.

c) The modified Watershed segmentation is used to expand the lung and the background by repeating the following loop until no more pixel can be added to the lung objects or the background.

i. Start with a dark threshold DT = GL (1) and a bright threshold BT = GL (3).

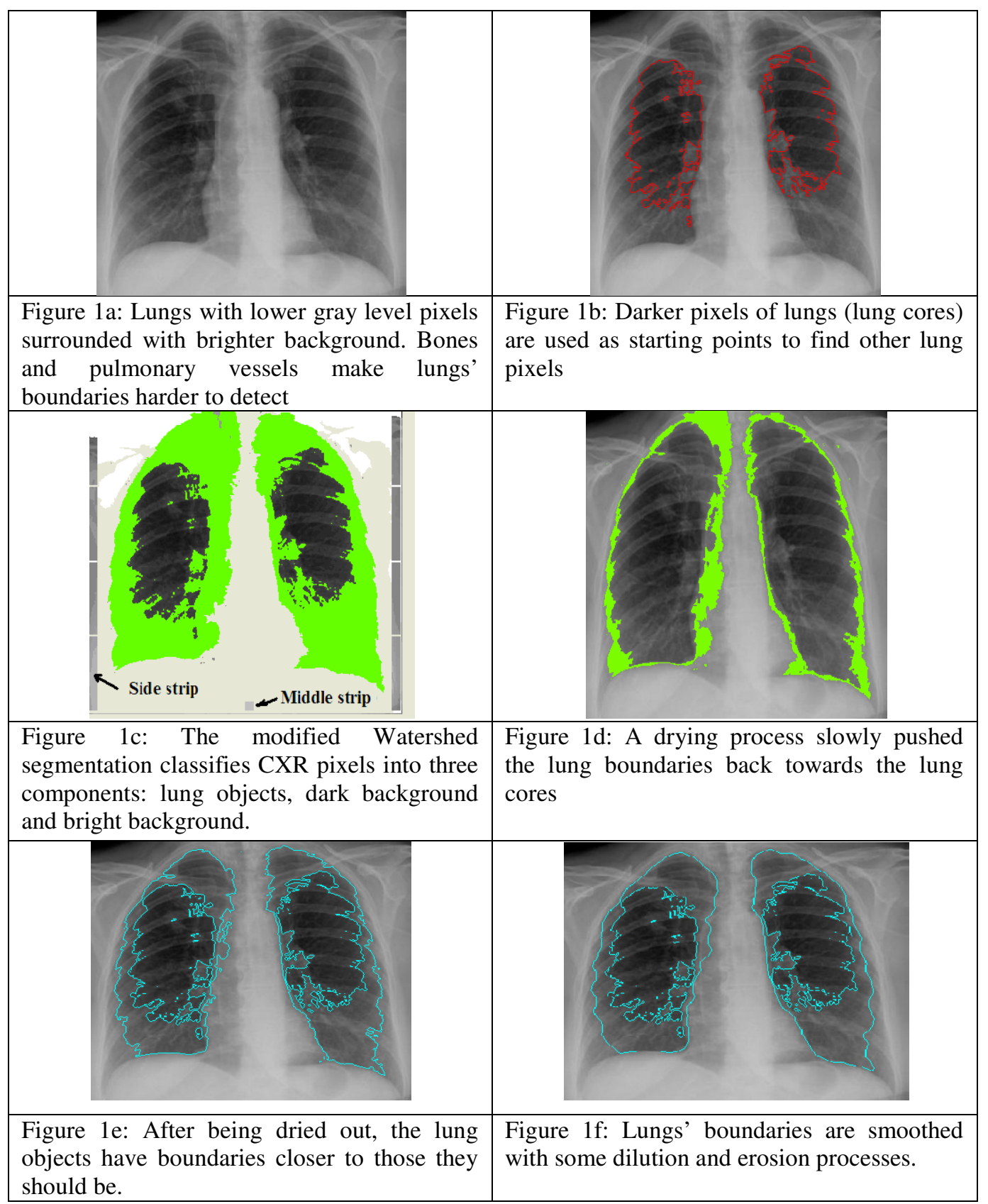

Figure 1: Chest X-ray image processing with a Watershed-based segmentation 
International Journal of Computer Science \& Information Technology (IJCSIT), Vol 3, No 2, April 2011

ii. In each iterative loop, slowly increment DT (max. 255) and decrement BT (min. 0) respectively.

iii. For all CXR pixels, conditionally mark unlabelled pixels as follows:

- If an unlabelled pixel that has a gray level lower than DT and is within theneighbourhood of pixels with labels $=+1$ (i.e. lung core pixels) or with labels $=+2$ (i.e. new lung object pixels), mark it with +2 .

- If an unlabelled pixel that has a gray level less than DT and is within the neighbourhood of pixels with labels $=-1$ (i.e. dark background pixels), mark it with -1 .

- If an unlabelled pixel that has a gray level higher than BT and is within the neighbourhood of pixels with labels $=-2$ (i.e. bright background pixels), mark it with -2 .

iv. Label all marked pixels with the values of their marks.

v. Repeat Steps ii, iii and iv, until no more pixel can be marked.

As a result, the modified Watershed segmentation classifies CXR pixels into three components: lung objects, dark background and bright background as illustrated in Figure $1 c$.

d) A slowly drying process, starting from a short vertical strip at the middle of the bottom part of CXR (Figure 1c), is applied with a gray level being decremented until it equals a low threshold. The threshold is calculated based on the average value (G1Av) of gray levels of added lung pixels (labelled with +2 ) and the average value $(\mathrm{G} 2 \mathrm{Av})$ of all CXR pixels. For example,

$$
\text { GLThreshold }=0.75 G 1 A v+0.25 G 2 A v
$$

Figure 1d illustrates the parts of expanded lung area being dried. The drying gray threshold needs more tuning for more accurate boundaries.

e) The boundaries of the dried lung objects (Figure 1e) are lastly smoothed by repeating some (say 5) dilution and erosion processes. The final result is illustrated in Figure 1f.

Once the lung objects have been detected, they can be analysed for abnormalities, some of them are presented in next sub-sections.

\subsection{Thoracic Diameter Measure}

An internal thoracic diameter is measured from the right atrial boundary of a heart to its left ventricle apex. The starting of a congestive heart failure can be detected when the cardiothoracic ratio becomes greater than $50 \%$ [6].

The thoracic diameter can be measure as follows.

\section{Thoracic Diameter Measurement Algorithm:}

a) Apply the "Lung Boundary Detection" algorithm to find the boundaries of the left and the right lungs.

b) Draw a rectangle enclosing each lung (called the lung box -Figures $2 \mathrm{a} \& \mathrm{~b}$ ). 
c) Start from the bottom edge of a lung box that has the bottom edge higher than that of the other. For example in Figure $2 \mathrm{a}$, the box on the right hand side (i.e. the box of the left lung) has the bottom edge higher. Normally the box of the right lung has a higher bottom edge (Figure $2 b$ ).

d) Detect the inner bottom corner of a lung (left lung -Figure 2a or right lung -Figure 2b). For example in Figure 2b, consider several pixels (e.g. 3) on the inner boundary of $t$ he right lung at some consecutive heights above the bottom edge of the lung box. The corner is detected when there is a sharp bend on the boundary.

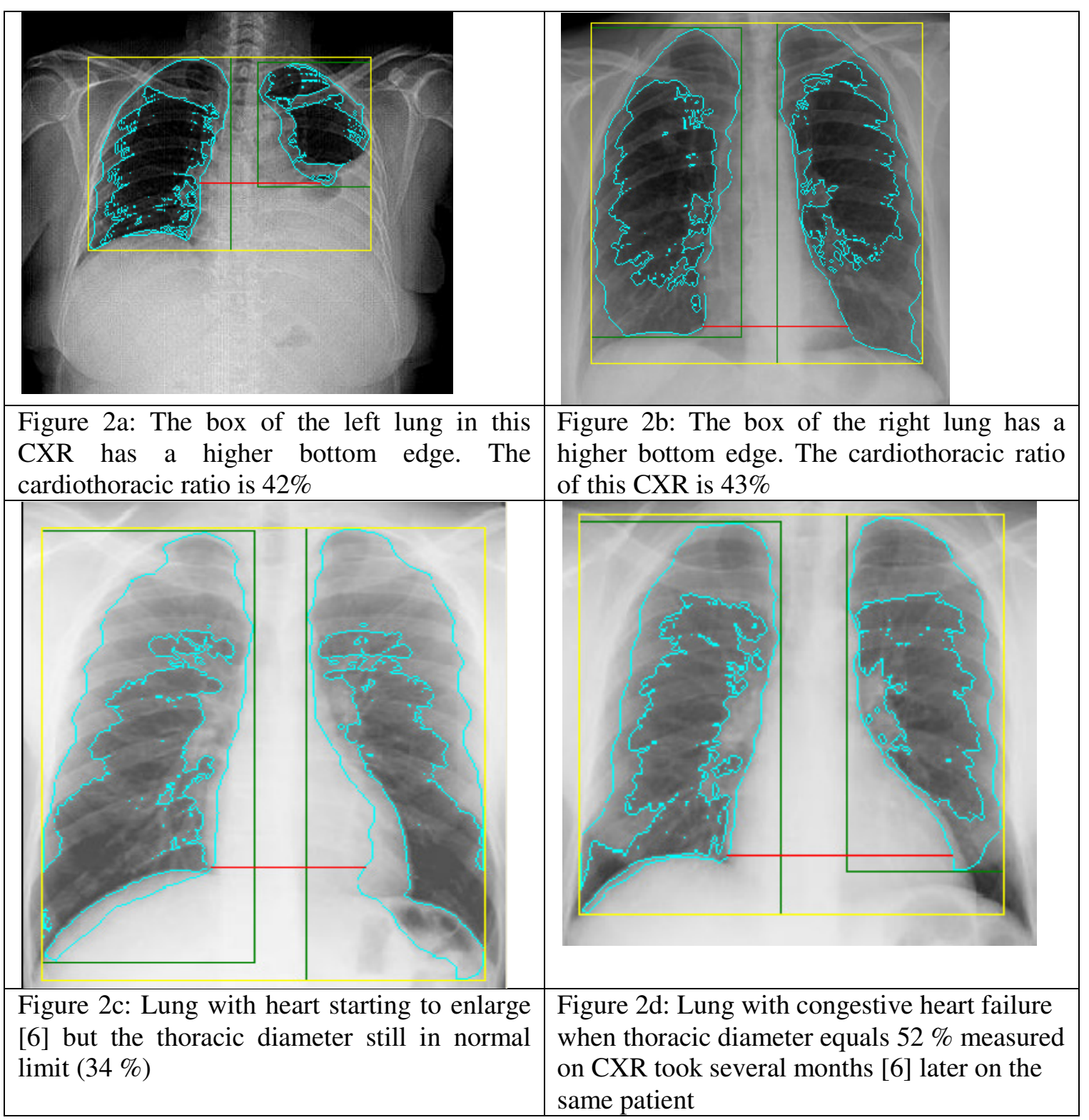

Figure 2: Measuring cardiothoracic ratio to detect congestive heart failure 
International Journal of Computer Science \& Information Technology (IJCSIT), Vol 3, No 2, April 2011

Table 1: Measurement of the cardiothoracic ratios on ten Chest X-rays.

\begin{tabular}{|c|c|c|c|c|c|c|c|c|c|c|}
\hline CXR & $\mathrm{X} 1$ & $\mathrm{X} 2$ & $\mathrm{X} 3$ & $\mathrm{X} 4$ & $\mathrm{X} 5$ & $\mathrm{X} 6$ & $\mathrm{X} 7$ & $\mathrm{X} 8$ & $\mathrm{X} 9$ & $\mathrm{X} 10$ \\
\hline $\begin{array}{l}\text { Cardiothoracic ratio } \\
(\%)\end{array}$ & 52 & 38 & 39 & 43 & 41 & 39 & 45 & 35 & 44 & 44 \\
\hline
\end{tabular}

e) From the corner, draw a horizontal segment to the closest boundary of the other lung (e.g. of the left lung in Figure 2b). The length of the segment is the thoracic diameter.

f) The cardiothoracic ratio is calculated as the percentage of the thoracic diameter compared to the internal diameter of the chest at the base of the common box of the two lungs.

Figures $2 c \& d$ illustrate two CXR images of the same patient, of which, Figure $2 d$ was taken several months after the other when the patient heart was detected being enlarged with the cardiothoracic ratio equal to $52 \%$. In the earlier CXR, the ratio is $34 \%$.

We measured the thoracic diameters on some other chest X-rays obtained from a hospital [7], and the result is tabulated in Table 1. In the table, the CXR X1 is of the patient with a heart failure mentioned above. The others are of patients without a heart failure, and their cardiothoracic ratios are all smaller than $46 \%$. Hence we can use this value as a threshold, and therefore, it is necessary to examine patients more regularly when their cardiothoracic ratios approaching a value higher than $46 \%$ to detect early symptoms of heart failure.

Heart failure patients often have their left lung volumes smaller than those of their right lungs. Hence the ratio of the two lung volumes of a patient can be considered as an additional feature for heart failure diagnosis. For normal lungs, this lung volume ratio should be about $100 \%$. On a CXR, a lung volume is estimated by counting the number of lung pixels. With the CXR of the heart failure patient (Figure 2d), the ratio between the volumes of the right and the left lungs is $126 \%$. A value of $120 \%$ is an estimated threshold for the lung volume ratio, which is under further investigation.

When the two thresholds for the cardiothoracic and the lung volume ratios are verified, it is very necessary to have closer examination for patients with the two ratios both higher than the thresholds.

The lung volume ratio alone is not a good feature for heart failure diagnosis. However, it can be used for diagnosis of lung collapse, which will be discussed in the next sub-section.

\subsection{Lung Collapse}

Lung collapse or atelectasis is a condition where the alveoli are deflated, as distinct from pulmonary consolidation, due to alveolar collapse or fluid consolidation. It may affect a part or the whole of one lung.

Lung collapse can be detected by checking the ratio of the right lung volume over that of the left lung. For normal lungs, this ratio is smaller than a high threshold (e.g. 140\%). For example, with the CXR in Figure 1, the ratio is $103 \%$. For the patient with a collapsed left lung (Figure $2 \mathrm{a}$ ); the ratio is $211 \%$. When the right lung is collapsed, the ratio will be smaller than a low threshold. We are investigating to find more accurate values for the high and the low thresholds of the lung volume ratios. 
When both two lungs are collapsed, the above lung volume ratio can not be used. However, the ratio of the total volume of the two lungs over the volume of the chest may be a good feature, which is under investigation. The ratio between the numbers of pixels of a lung and its lung box is also a good feature for CXR diagnosis, and need further investigation. For example, with the lungs in Figure $2 \mathrm{a}$, the ratio for the right lung is $64 \%$ and the left lung is $59 \%$. For the lungs in Figure 1, the ratios are $70 \%$ and $63 \%$ respectively. However, for the lungs in Figure $2 \mathrm{~d}$, the ratios are $58 \%$ and $64 \%$.

The ratio between the number of pixels of a lung core and that of the lung is also a feature for CXR diagnosis.

\subsection{Nodule Detection}

Most cancer and tuberculosis cases start with the appearance of small nodules, which can be benign or malignant with malignant nodules growing up quicker. Nodule pixels are often brighter than the surrounding areas, especially calcified parts, but in some cases, the difference in gray levels is not significant. Furthermore, ribs and pulmonary arteries, which often have higher gray levels, also contribute to the complexity of lung tissue and make some nodules being undetectable. In up to $30 \%$ of cases, nodules are overlooked by radiologists on their first examinations [8], although they are visible in retrospect, especially when computer-aided diagnostic tools are used to focus radiologists' attention on suspected areas [3].

Early cancer and tuberculosis nodules have the following characterises:

- Nodule pixels are brighter than surrounding pixels (i.e. higher gray level)

- Their areas are smaller than a high threshold (because they are early nodules) but greater a low threshold (to differentiate them from noisy areas)

We proposed a method to detect early nodules as follows [9].

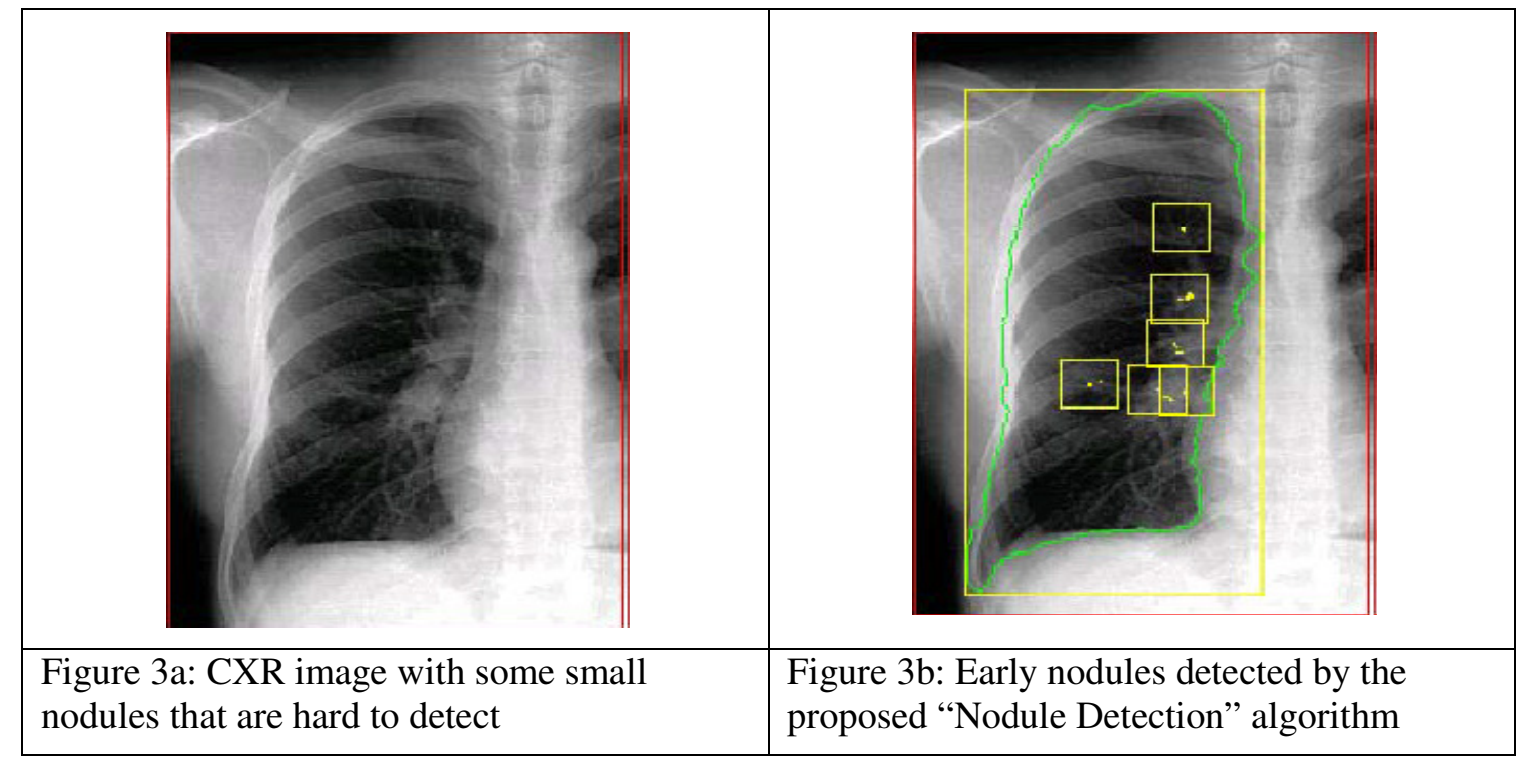

Figure 3: Detection of nodules, which are difficult to detect due to the presence of ribs and pulmonary arteries 
International Journal of Computer Science \& Information Technology (IJCSIT), Vol 3, No 2, April 2011

\section{Nodule Detection Algorithm:}

a) Detect lung objects: Apply the "Lung Boundary Detection" algorithm to find the boundaries of the left and the right lung. Now pay attention to one lung, e.g., the right lung (Figure 3a). Repeat the following steps for all pixels of the lung.

b) Find brighter lung pixels within a scanning window:

- Apply a small fixed size window -called scanning window- to every pixel inside a lung object, which has not been marked as part of suspected nodules, and has the gray level higher than a threshold. This level is selected based on the gray level histogram of the whole the CXR, e.g. GL(1).

- Find the average and the maximal gray levels of the pixels within the scanning window. Select a local gray-level threshold between the average and the maximal levels. Mark all pixels that have gray levels higher than the local gray-level threshold.

c) Detect early nodule pixels within the scanning windows: Count the number of pixels that have gray levels higher than the local threshold. If the counted number is within the specific range of a low and a high thresholds then mark the pixel as one of a suspected nodule.

Figure $3 \mathrm{~b}$ shows the result obtained with the above algorithm. We are tuning the algorithm with 100 CXR images collected from hospitals [7, 10] based on the judgement of medical professionals $[7,11]$.

\subsection{CXR Computation and Examination}

CXR obtained from hospitals are often with a very big size (about 2800 by 2300 pixels), which can not be displayed the whole on a normal desktop screen without being resized or zoomed. To reduce computing time, we resized them to a smaller size (about 640 by 640 pixels). When a CXR have been processed, suspected areas are marked. Examiners can click on any area to get a zoom-in with more details as illustrated in Figure 4.

We are tuning our CAD tool for CXR analysis with 100 CXR obtained from hospitals $[7,10]$. The designed CAD tool with CXR examination facilities will be supplied to medical professionals to facilitate their judgment.

\section{CONCLUSION}

This paper presents some basic methods for automated CXR analysis, which may be used in a $\mathrm{CAD}$ tool. The experimental results obtained with the proposed algorithms to detect early nodules for lung cancer and TB, as well as lung collapse, congestive heart failure are very encouraging. Further tuning is in progress. Works for other chest diseases are in examination.

\section{ACKNOWLEDGEMENT}

The work reported in this paper is a continuing work at the Faculty of Information Sciences and Engineering, University of Canberra. 
The author is grateful to Dr. Peter Nickolls (Prince of Wales Medical Research Institute, New South Wales), Dr Warwick Lee (Bowral and District Hospital, New South Wales), Dr. NgocThach Tran (Tuberculosis Hospital, Saigon) and Dr. Quoc-Truc Nguyen (Ulcer and Cancer Hospital, Saigon) for their medical advice and X-ray images supply. Medical information collected from some medical Web sites $[6,12]$ is also acknowledged.

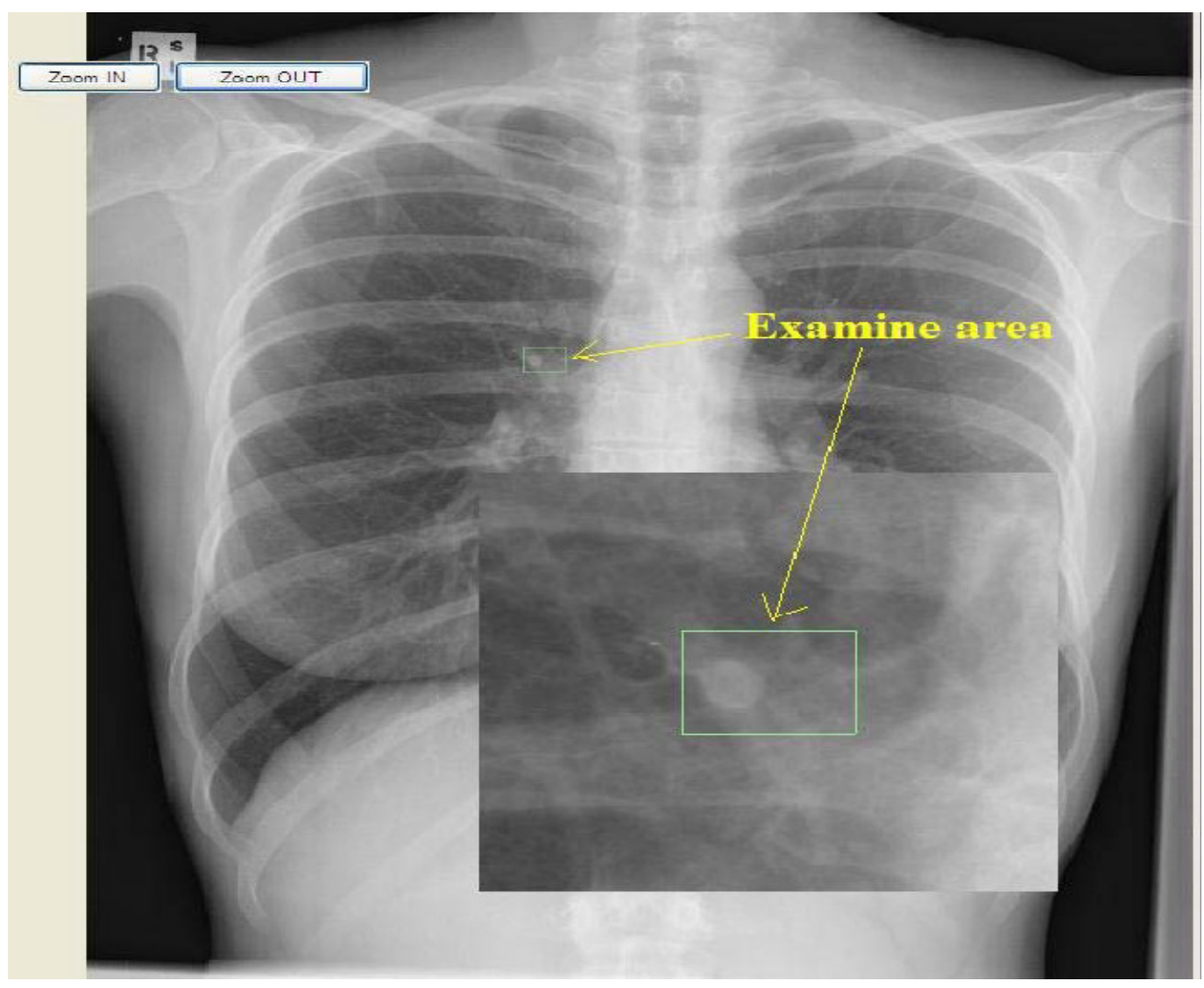

Figure 4: CXR examination screen with a suspected area being display with details.

\section{REFERENCE}

[1] Le, K, (2011) "Chest X-Ray Analysis for Computer-Aided Diagnostic", First International Conference on Computer Science and Information Technology, CCSIT 2011, Bangalore, India January 2-4, 2011, Proceedings, Part III, pp.300-309.

[2] Gurcan, M.N., et al., (2002) "Lung nodule detection on thoracic computed tomography images: preliminary evaluation of a computer-aided diagnosis system", Med Phys, 29(11): 2552-8.

[3] Kakeda, S. et al., (2004) "Improved Detection of Lung Nodules on Chest Radiographs Using a Commercial Computer-Aided Diagnosis System", American Journal of Roentgenology, 182, February, pp. 505-510. 
International Journal of Computer Science \& Information Technology (IJCSIT), Vol 3, No 2, April 2011

[4] Beucher, S. and Meyer, F., (1992) “The Morphological Approach of Segmentation: The Watershed Transformation," Mathematical Morphology in Image Processing, E. Dougherty, ed., pp. 43-481, New York: Marcel Dekker.

[5] Nguyen, H.T. el al., (2003) "Watersnakes: Energy-Driven Watershed Segmentation", IEEE Transactions On Pattern Analysis and Machine Intelligence, Vol. 25, No. 3, March, pp.330-340.

[6] Spencer, B. et al., "Introduction to Chest Radiology", University of Virginia Health Sciences Center, Department of Radiology,

http://www.med- ed.virginia.edu/courses/rad/cxr/index.html

[7] Lee, Warwick, (2008) (Bowral and District Hospital, New South Wales), private correspondence.

[8] Suzuki K., et al., (2005) "False-positive Reduction in Computer-aided Diagnostic Scheme for Detecting

Nodules in Chest Radiographs by Means of Massive Training Artificial Neural Network", Academic

Radiology, 12, No 2, February, pp. 191-201.

[9] Le, K, (2006) "Lung X-Ray Image Analysis for Automated Detection of Early Cancer and Tuberculosis", WSEAS Transactions on Information Science and Applications, Issue 12, Volume 3, December, pp. 2347-2354.

[10] Tran, N.T. (2008) (Pham Ngoc Thach Hospital, Ho-Chi-Minh City), Private correspondence.

[11]Nickolls P (2006) (Prince of Wales Medical Research Institute, NSW), Private correspondence.

[12]MEDPIX, http://rad.usuhs.mil/medpix/medpix.html 